\title{
An Adaptive Contention Window Scheme for 802.11ah WLANs
}

\author{
Renzheng $\mathrm{Gao}^{1}$, Xiaoying $\mathrm{Lei}^{1}$, and Qiang $\mathrm{Hu}^{1}$ \\ ${ }^{1}$ College of Information Enginnering Yangzhou University, Yangzhou 225127, China
}

\begin{abstract}
In order to cope with the rapid development of the Internet of things, IEEE working group has proposed a new wireless network technology, 802.11ah. IEEE 802.11 ah operates at sub $1 \mathrm{GHz}$ band, and can provide long transmission range, wide coverage, while supporting more than 6,000 of station to be connected. In order to alleviate the high collision probability, 802.11ah MAC protocol involves in the RAW (restricted access window) mechanism combined with grouping mechanism. However, in 802.11 ah WLAN (wireless local area network), most of the stations operate at sleep mode, and it is expected that at the start of a BI, the network suffers much higher collision probability than at the latter period of the BI. In order to improve these drawbacks, we propose an adaptive contention window scheme based on which an optimum contention window size is adapted at the start of the BI, and then gradually halved when the transmission completes successfully. Through conducting simulations, we prove that our proposed protocol can enhance the performance of 802.11ah WLAN.
\end{abstract}

\section{Introduction}

The Internet of things (IoT) is an important part of the new generation of information technology, and it is also an important stage of development in the "information" era. IoT describes a vision where everyday objects can be equipped with identifying, sensing, networking and processing capabilities that allow them to communicate with one another over the Internet [1]. Nowadays, IoT has been used in the major developed countries in the world, but the global system of networking industry is still in the process of establishing and perfecting. In order to provide ubiquitous connectivity, the IoT (Internet of Things) requires wireless network technologies which can provide high performances even in a dense network environment.

The IEEE 802.11ah working group has proposed a WLAN (wireless local area network) technology that can work on unlicensed frequency bands below $1 \mathrm{GHz}$. Due to the advantages of low frequency propagation, 802.11ah WLAN (wireless local area network) technology can achieve a wide range of network transmission function, and accommodates multiple stations [2]. The 802.11 ah MAC (media access control) proposes an RAW (restrict access window) mechanism working under the support of a grouping scheme. This protocol can provide long transmission range, wide coverage, and support more than 6,000 of

Corresponding author: xylei@yzu.edu.cn 
stations to be connected, and applies packet technology, it assumes that each group of stations has the same number, and takes BI (beacon interval) as the cycle to carry out data transmission. Here is the process: during a beacon interval (BI), AP initiates several restricted access windows (RAWs), and an RAW is sliced into equal time slots which can be allocated to single station or a group of stations. Stations contend to access channel according to the allocated RAW [3].

However, the 802.11ah MAC layer is still based on the 802.11DCF (distributed coordination function) protocol. And it is proved that the legacy DCF (distributed coordination function) protocol raises severe channel contention and hidden terminal problems in large size networks. On the other hand, most of the stations in 802.11ah operate under PSM (power saving mode), according to which the stations wake up periodically to transmit packets, and then enter into sleep mode. It can be expected that, there are more contending stations at the start of BI than in the later period, therefore, these collisions will cause the waste of channel resources and the consumption of energy.

In order to improve $802.11 \mathrm{ah}$, we propose an adaptive contention window scheme, based on which the initial value of the current contention window is set according to the number of competing stations, and with the successful transmission, the value of contention window is reduced, or else the contention window size sustains to be the current value in case of collision occurs. More details will be present in Section 3.

The rest of the article is organized as follows: Section 2 provides an overview of the IEEE 802.11ah, IEEE 802.11DCF and the related works of this paper while Section 3 presents the details of the proposed scheme. Section 4 conducts the discrete simulations to evaluate the performance of proposed scheme, and finally, section 5 draws conclusion remarks.

\section{Preliminaries}

\subsection{IEEE 802.11DCF}

Figure 1 illustrates the IEEE 802.11 DCF scheme [9]. In the basic DCF, we choose two stations as sender and receiver, first, the sender should sense the channel for a period of DIFS (DCF inter frame space) before its transmission. If the channel remains idle for DIFS (DCF inter frame space), the station can access the channel to transmission. Otherwise, the station starts a backoff process by randomly selecting a number from $[0, \mathrm{w}]$ as the value of the $\mathrm{BC}$ (backoff counter), where $\mathrm{w}$ is the value of the current contention window of this station. The 802.11standard has defined the maximum and minimum values of contention windows (CWmin, CWmax). The initial value of $\mathrm{w}$ is $\mathrm{CWmin}$, it doubles in case the station encounters a collision, otherwise, if a transmission is successful, the value will be set back to CWmin. On the other hand, when the value of $\mathrm{w}$ reaches to CWmax, the station has to sustain it even if it encounters another collision. However, when the number of collisions of the station is too high to reach the upper limit, we will discard the packet that the station needs to transmit, and it must reselect the new BC to compete channel with others. While staying in backoff procedure, the station continues to sense the channel, and as soon as an idle channel is sensed, the station reduces its $\mathrm{BC}$ for one time slot. When the $\mathrm{BC}$ of the station reaches to 0 , the station can access to the channel and start its transmission immediately. When the sender transmits data successful, a SIFS (short inter frame space) later, the receiver returns a reply packet and the sender catch it. At this point, the entire data transmission between the stations is completed. 


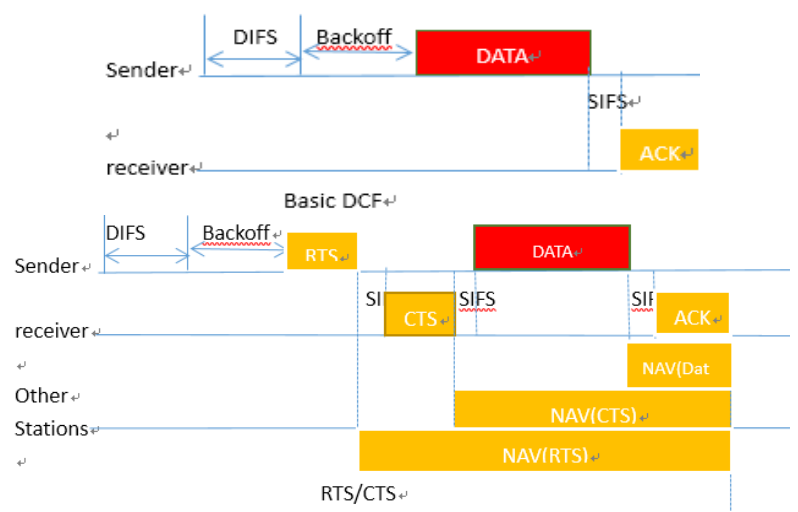

Fig. 1. IEEE 802.11 DCF.

In addition to the basic access, an optional four way hand-shaking technique, known as request-to-send / clear-to-send (RTS/CTS) mechanism has been standardized. Before transmitting a packet, a station operating in RTS/CTS mode "reserves" the channel by sending a special Request-To-Send short frame. The destination station acknowledges the receipt of an RTS frame by sending back a Clear-To-Send frame, after which normal packet transmission and ACK response occurs. Since collision may occur only on the RTS frame, and it is detected by the lack of CTS response, the RTS/CTS mechanism allows to increase the system performance by reducing the duration of a collision when long messages are transmitted. As an important side effect, the RTS/CTS scheme designed in the 802.11 protocol is suited to combat the so-called problem of hidden terminals, which occurs when pairs of stations result to be unable to hear each other.

In Figure 1, a sender that wants to transmit a packet, waits until the channel is sensed idle for a DIFS, follows the backoff rules explained above, and then, instead of the packet, preliminarily transmits a special short frame called request to send (RTS). When the receiver detects an RTS frame, it responds, after a SIFS, with a clear to send (CTS) frame. The transmitting station is allowed to transmit its packet only if the CTS frame is correctly received. The frames RTS and CTS carry the information of the length of the packet to be transmitted. This information can be read by any listening station, which is then able to update a network allocation vector (NAV) containing the information of the period of time in which the channel will remain busy. Therefore, when a station is hidden from either the transmitting or the receiving station, by detecting just one frame among the RTS and CTS frames, it can suitably delay further transmission, and thus avoid collision.

\subsection{IEEE 802.11 AH}

The 802.11ah TGah (Task Group) has started establishing the IEEE 802.11ah amendment since November 2010, which is completed in March 2016[4]. Comparing with the existing IEEE 802.11 WLAN (wireless local area network) technology, 802.11ah revision involves many unique features in the PHY (physical) protocol and MAC (media access control) protocol.

IEEE 802.11ah physical layer reuse a series of 802.11ac technology [5] and make it improved so that it can work in the S1G unlicensed band. As shown in Figure 2, the 802.11ah of the channel definition is different in various countries: e.g., the United States, South Korea, 902-928 MHz; 917.5-923.5 MHz; 863-868 MHz; Chinese, Europe, Japan, 755-787 MHz; 916.5-927.5 MHz [6]. Singapore uses two independent available frequency bands, 866-869 MHz and 920-925 MHz. The PHY layer adopts OFDM (orthogonal frequency division multiplexing) and works at $1 \mathrm{GHz}$ frequency, which is 10 times lower 
than the main frequency version. The proposed channel bandwidth includes $1 \mathrm{MHz}, 2 \mathrm{MHz}$, $4 \mathrm{MHz}, 8 \mathrm{MHz}$ and $16 \mathrm{MHz}$, of which $1 \mathrm{MHz}$ and $2 \mathrm{MHz}$ channel bandwidth are widely adopted in many countries [7].

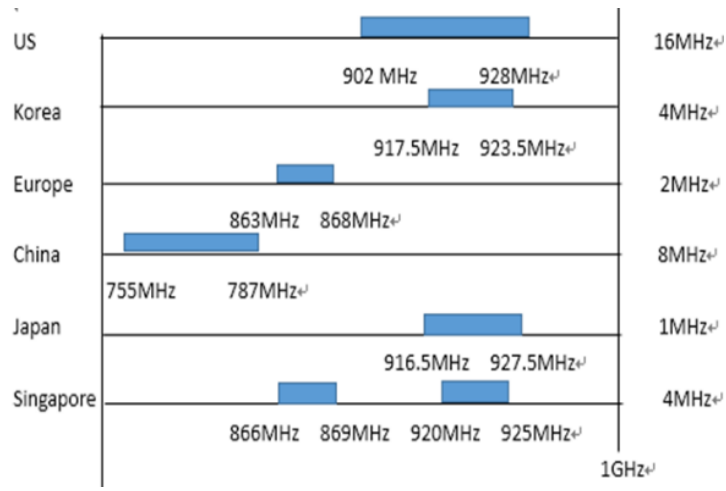

Fig. 2. IEEE 802.11ah Global channel allocation.

Because the IEEE802.11 MAC protocol uses 802.11 DCF (distributed coordination function), it can cause large packet collision probability and trigger hidden stations problems in large size networks. The IEEE 802.11ah MAC proposes to an RAW (restrict access window) scheme. The core idea is that a BI (beacon interval) is divided into equal time slots, named, RAW, and each of the time slots can be assigned to a station or a group of stations [8]. AP stores RAW relevant information into the beacon frame, including the starting time, length, the number of time slot and the AID of stations that can be allowed to transmit in each time slot. The information to the initiated RAWs is broadcasted through short Beacon frames by the AP. A station can access channel during the allocated RAW, following the process defined by DCF protocol.

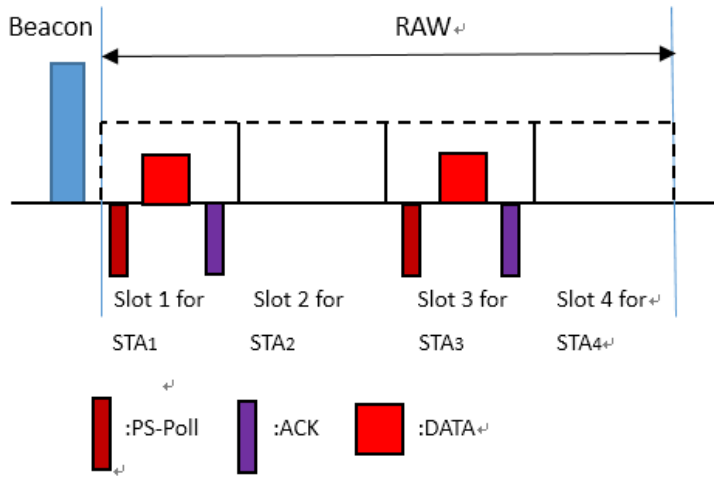

Fig. 3. RAW based channel access.

Figure 3 depicts a RAW-based media access approach. In a BI, AP allocates the time slots to 4 stations, STA1, STA2, STA3 and STA4, STA1 and STA3 are in active mode, while STA2 and STA4 are in doze mode. STA1 and STA3 by sending PS-Poll frames tell the AP that the station is in active mode. The AP receives the station information and to assign time slots to stations, at the same time, the station starts listening beacon frame, which carry the relevant information about RAW, including the beginning time of RAW, time length of RAW, the number of Slot in the RAW and which RAW will be allocated to the transmission, etc. When the STA1 and STA3 receive this information, they will know that the AP is allocated to their access time, Slot1 for STA1 and Slot2 for STA3. Then, the 
stations access the channel within the time slot assigned to each other and carry out data transmission. This mechanism reduces the number of devices trying to access channel at a given time period, and thus alleviates the channel contention.

However, the above mechanism does not guarantee effective channel utilization. No matter what the state of the device is, the AP will allocate the time slot for them, in case the station is in sleeping mode, the allocated time slot will be wasted. In order to improve the efficiency of channel utilization, many scholars have studied the protocol in depth, and proposed a series of effective improvements, such as the introduction of RA (resource allocation) frames in 802.11ah improving the problem of the allocated time slot of the dormant stations, and 802.11ah uses the TWT (target wake-up time) mechanism, which allows AP to manage the wake-up time of the stations below BSS, the stations can be in different time periods to wake up, thereby reducing competition conflict, improve the efficiency of the system, but also greatly reduce the power consumption of the stations. On this basis, we will also do more research on this problem in the future.

\subsection{Related works}

However, the random backoff method can encounter collisions, stations can choose a same $\mathrm{BC}$ value because they have no idea of the $\mathrm{BC}$ selected by others. Previous works have presented different approaches for solving this problem [10-14]. [10] proposed station can reserve a $\mathrm{BC}$ value through the data packet being transferred and store the reserved $\mathrm{BC}$ into the packet header, other stations can monitor this packet and know the value of $\mathrm{BC}$ according to the broadcast characteristics based on wireless networks; [11] is suggested that when a station accesses the channel successfully, it can reuse its BC by increasing the value of $\mathrm{M}$. Here, $\mathrm{M}$ is the number of stations that belong to the same BSS (basic service set), this station can continue to occupy channel and transmit data packet, without choosing a new $\mathrm{BC}$ to compete channel; [12], according to this scheme, when a station wants to transmit a packet, it stores the next backoff counter to be used in the MAC header of current packet.

The other stations which overhear this packet avoid choosing the same backoff counter. This reservation-based scheme successfully reduces the collision probability in certain level while increasing whole network throughput; In [13] a virtual backoff algorithm is proposed. When multiple stations select an identical BC, the channel accesses of these stations are rearranged according to their sequence numbers, in other words, the stations that choose the same $\mathrm{BC}$ are made out of the sequence numbers in the virtual space, and these serial numbers are used to access the channel, it can effectively relieve collisions in this way; [14]design a collision-free backoff method to improve the backoff function, that enable the stations to reserve their own BCs using previously delivered control packets, after these stations complete their data transmissions they release the reserved BCs to stations having not reserved a $\mathrm{BC}$.

These approaches still suffer some drawbacks: when a station chooses the value of $\mathrm{BC}$ randomly in [10], the randomly selected $\mathrm{BC}$ may be with a large value, thus brings in long access delay and waste of resources; in [11], the number of stations contending for channel can be much less than $\mathrm{M}$, when the $\mathrm{M}$ value is far greater than the number of stations, the scheme is meaningless; in[12], when there a hidden station in the network, other stations may cause collisions because they cannot monitor its reservation $\mathrm{BC}$; [13] requires for hardware modification, the realization of which is complicated, which is not operable in the process of experiment; in [14], we assume that each station sends a packet and can be monitored by other stations, that may fail if all the stations work in CAM (continual active mode), because the control packet transmission will not occur, thus, the $\mathrm{BC}$ reservation process cannot be activated. If in this case, we will do more research on it in our future work. 


\section{Adaptive contention window scheme}

In this section, we design an adaptive contention window method to improve the performance of IEEE 802.11ah. Our proposal is based on the following observations: In 802.11PSM (power saving mode), stations periodically wake up according to BI (beacon interval), it is expected that at the start of a BI, there are much more active stations, which can bring in a high collision probability. And with the passing of BI, the number of active stations decreases, thus the channel competition eliminates. Furthermore, RAW scheme is supported by a grouping mechanism, therefore, it's reasonable to obtain the number of stations per group.

In proposed scheme, instead of utilizing a minimum contention window at the beginning of the backoff process and doubles it each time a collision occurs, we propose to begin with an optimum contention window, CWopt. And if the stations transmission completes successfully, the value of the CW decreases to half, or else with collision occurs, the value of the CW sustains. The value of CWopt is provided in [9] as follows:

$$
C W_{o p t} \approx N \sqrt{ }(2 T c)
$$

Here, $N$ is the number of stations in the network, $T c$ is the average time to detect the collision of each station, which can be obtained according to (2):

$$
T c=H+E[P]+D I F S+\delta
$$

where $H$ is the packet header, the value of which equals to

$$
H=P H Y h d r+M A C h d r
$$

PHYhdr is the header of physical layer and MAChdr is the header of MAC (media access control) layer. $\mathrm{E}[\mathrm{P}]$ is the average length of the longest packet payload involved in a collision, and $\delta$ is the propagation delay time.

Since 802.11 ah is based on a grouping mechanism, we assume there are Nt stations in the network, and they are divided into $\mathrm{G}$ groups then the number of stations in each group is $\mathrm{Nt} / \mathrm{G}=\mathrm{n}$, according to (1), The optimum $\mathrm{CW}$ applied by stations at the beginning of backoff process is $\mathrm{Nt} / \mathrm{G} * \sqrt{ }(2 \mathrm{Tc})$, and for each of successful transmission, this value reduces to $1 / 2$, otherwise it sustains. In this way, at the start of a $\mathrm{BI}$, the stations contend channel with a large window size, which can effectively reduce the collision probability. And as more and more stations complete their transmissions and enter into sleep mode, a smaller value is applied as contention window, which can reduce the channel access delay and improve network transmission performance.

\section{Performance evaluation}

\subsection{Simulation environment}

OPNET is an excellent network simulation and modeling tool. It supports object-oriented modeling and provides a graphical editing interface that is more convenient for users to use. The powerful capabilities and comprehensiveness provided by OPNET can almost simulate any network device and support various network technologies. In addition to the ability to simulate fixed communication models, OPNET's wireless modeler can also be used to build models of packet radio networks and satellite communication networks. At the same time, OPNET has outstanding performance in the design of the new network and the analysis of the existing network. It provides the same environment as the real network for the research 
of communication protocols and algorithms, and the perfect result analyzer provides an effective and intuitive tool for network performance analysis.

In this paper, we mainly use its WLAN (wireless local area networks) environment model, and based on this model, the network environment is modified as the experimental model we need. First, we are modifying the protocol to $802.11 \mathrm{ah}$, including the width of transmission channel and the data transmission rate, on the other hand, we modify the backoff algorithm for our proposed scheme.

Figure 4 depicts the transmission process model of WLAN including 10 working modules. INIT: Initialization of the process model, BSS_INIT: Schedule a self interrupt to wait for mac interface, IDLE: The purpose of this state is to wait until the packet has arrived from the higher or lower layer, FRM_END: The purpose of this state is to determine the next unforced state after completing transmission, WAIT_FOR_RESPONSE: The purpose of this state is to wait for the response after transmission. The only frames which require acknowledgements are RTS and DATA frame, DEFER: This state defer until the medium is available for transmission, BKOFF_NEEDED: In this state we determine whether a back-off is necessary for the frame we are trying to transmit, BACKOFF: Processing Random Backoff, TRANSMIT: In this state we complete data transmission, SCAN: Record the state name if running in debug mode. The proposed scheme is to modify the backoff algorithm in the BACKOFF working module.

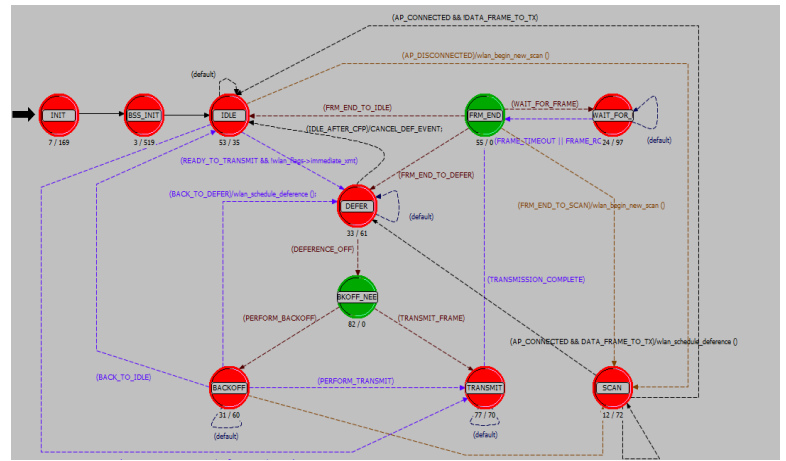

Fig. 4. WLAN process model.

\subsection{Results}

In this section, we evaluate the performance of the proposed scheme using the OPNET simulator. The network is composed of a AP and several stations, and the number of stations is varied to evaluate the performance of the scheme comparing with the legacy protocol. We select the number of stations as $60,80,100,120$ and 140 , and then create network work environment for them, simulate these projects. We compare these projects with the results of the simulation. All stations send or receive packets periodically. More details of the parameters are shown in the Table. 1.

Table 1. Simulation parameters

\begin{tabular}{|l|l|}
\hline Physical Characteristics & OFDM(802.11a) \\
\hline Data Rates(bps) & $2.6 \mathrm{Mbps}$ \\
\hline Channel setting & Channel 1 \\
\hline Transmit power(W) & 0.05 \\
\hline
\end{tabular}




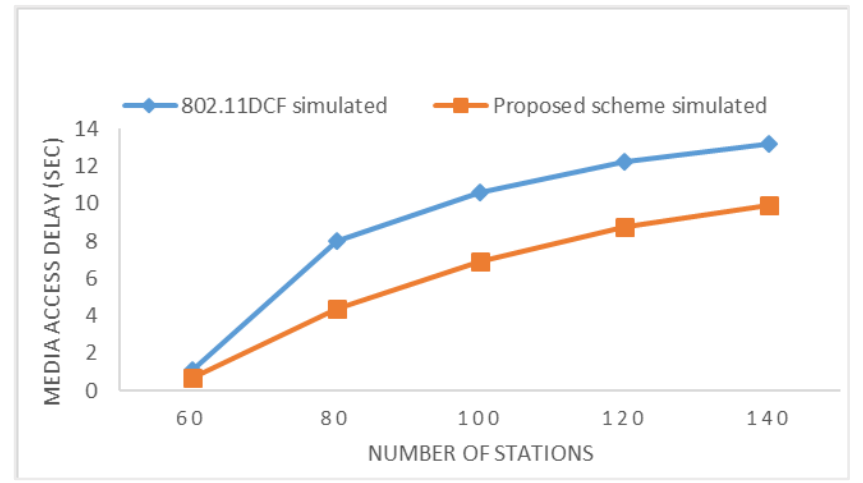

Fig. 5. Media access delay (sec).

Figure 5 describes the obtained media access delay as varying the number of stations under the legacy scheme as well as the proposed scheme. We can find that when the number of stations increases, the media access delay increases gradually, but the proposed scheme significantly reduces the media access delay. It is because by applying an adaptive contention window size, proposed protocol can reduce the time consumed in backoff process, thus decrease channel access time.

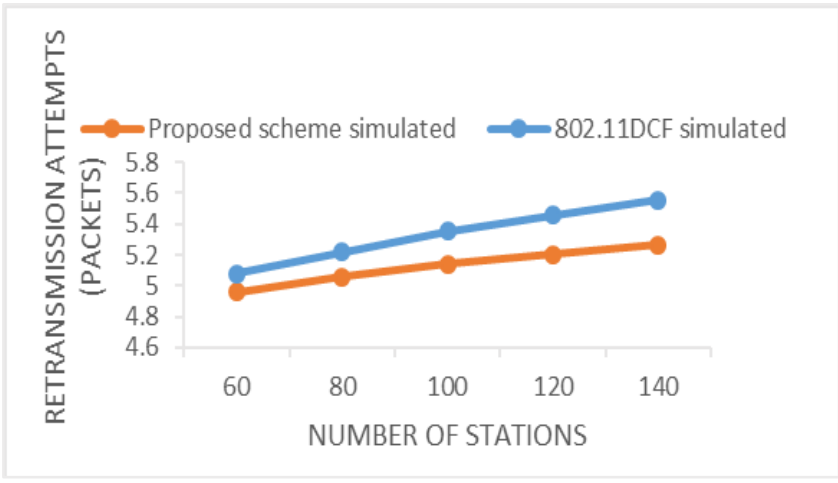

Fig. 6. Retransmission attempts (packets).

Figure 6 depicts the transformation of the retransmission attempts as varying the number of stations of the whole network. We can find that with the number of stations increasing, the network retransmission attempts grow up in both scheme, but in a lower level in proposed scheme. It is because by adapting the contention window size, our protocol can reduce the collision probability, thus bring in high performance.

\section{Conclusion}

Based on the fact that there are more contending stations at the start of a BI then in later period, we have proposed an adaptive contention window scheme to improve the performance of $802.11 \mathrm{ah}$. Instead of utilizing a minimum contention window at the start of a BI and doubles it when collision occurs, we utilize an optimum contention window at the start. And sustains its value in case of collision, or else reduces it to be half of its previous value for success transmission. By conducting simulations, we confirmed that the proposed scheme can effectively reduce the media access delay and the network retransmission attempts, improving the overall performance of the network. 


\section{References}

1. Xiaoying Lei,Seung Hyong Rhee. "Performance Improvement of Sub 1 GHz WLANs for Future IoT Environments",(2017).

2. BiKaichun, Xia Wan Li, Li Weina. "Foreign networking perspective. Electronics Industry Press",(2014).

3. Bel A. Adame T. Bellalta B. Barcelo J. Gonzalez J., and Oliver M "CAS-based channel access protocol for IEEE 802.11ah WLANs", European Wireless 2014, 20th European Wireless Conference, (2014).

4. J.-O Seo, C. Nam, S.-G. Yoon, and S. Bah"Group-based contention in IEEE 802.11ah networks, "IEEE, (2014);

5. Ji B., Song K., H u Y., and Chen H., "Cooperative Transmission Mechanisms in Next Generation WiFi: IEEE 802.11ac," International Journal of Distributed Sensor Networks, vol. 2014, (2014).

6. Aust S., Prasad R.V., and Niemegeers I.G., "IEEE 802.11ah: Advantages in standards and further challenges for sub $1 \mathrm{GHz}$ Wi-Fi," In Proceedings of IEEE International Conference on Communications (ICC), IEEE, June (2012).

7. Khorov E., Lyakhov A., Krotov A. and Guschin A., "A survey on IEEE 802.11ah: An enabling networking technology for smart cities," Computer Communications, vol. 11, pp.53-69,(2015).

8. Hazmi A., Rinne J., and Valkama M., "Feasibility study of IEEE 802.11 ah radio technology for IoT and M2M use cases," In Globecom Workshops (GC Wkshps), IEEE, (2012).

9. Bianchi G., "Performance analysis of the IEEE 802.11 distributed coordination function," IEEE Journal on Selected Areas in Communications, vol.18, no.3, pp. 535-547, (2000)

10. J. Choi, J. Yoo, S. Choi, and C. Kim, "EBA: An enhancement of the IEEE 802.11 DCF via distributed reservation," IEEE Trans. Mobile Comput., vol. 4, pp. 378-390, (2005).

11. Y. He, J. Sun, X. Ma, AV. Vasilakos, R. Yuan, W. Gong, "Semi-random backoff: towards resource reservation for channel access in wireless LANs," IEEE/ACM Trans. Netw. (TON), vol.21, no.1, pp.204-217,(2013).

12. Choi, J., Yoo, J., Choi, S., \& Kim, C. EBA: An enhancement of the IEEE 802.11 DCF via distributed reservation. IEEE Transactions on Mobile Computing, 4, 378-390, (2005).

13. P.V. Krishna, S. Misra, M.S. Obaidat, V. Saritha, "Virtual backoff algorithm: An enhancement to 802.11 medium-access control to improve the performance of wireless networks," IEEE Trans. On Vehicular Tech., vol.59, no. 3, pp.1068-1075,( 2010).

14. Xiaoying Lei Yangzhou, ChinaSeung Hyong Rhee"Design of a Collision-free Backoff Method to Improve the IEEE 802.11 DCF ",(2016). 